\title{
Increased resistance of a methicillin-resistant Staphylococcus aureus $\Delta$ agr mutant with modified control in fatty acid metabolism
}

\author{
Hun-Suk Song \\ Konkuk University \\ Tae-Rim Choi \\ Konkuk University \\ Yeong-Hoon Han \\ Konkuk University \\ Ye-Lim Park \\ Konkuk University \\ Jun Young Park \\ Konkuk University \\ Soo-Yeon Yang \\ Konkuk University \\ Shashi Kant Bhatia \\ Konkuk University \\ Ranjit Gurav \\ Konkuk University \\ Yun-Gon Kim \\ Soongsil University \\ Jae-Seok Kim \\ Hallym University College of Medicine \\ Hwang-Soo Joo \\ Duksung Women's University \\ Yung-hun Yang ( $\sim$ seokor@konkuk.ac.kr) \\ Konkuk University
}

Original article

Keywords: MRSA, $\beta$-lactam antibiotic, biofilm, fatty acid, surfactant

Posted Date: March 30th, 2020

DOI: https://doi.org/10.21203/rs.2.20153/v2 
License: (c) (i) This work is licensed under a Creative Commons Attribution 4.0 International License. Read Full License

Version of Record: A version of this preprint was published at AMB Express on April 7th, 2020. See the published version at https://doi.org/10.1186/s13568-020-01000-y. 


\section{Abstract}

Methicillin-resistant Staphylococcus aureus (MRSA) strains are distinct from general Staphylococcus strains with respect to the composition of the membrane, ability to form a thicker biofilm, and, importantly, ability to modify the target of antibiotics to evade their activity. The agr gene is an accessory global regulator of gram-positive bacteria that governs virulence or resistant mechanisms and therefore an important target for the control of resistant strains. However, the mechanism by which agrimpacts resistance to $\beta$-lactam antibiotics remains unclear. In the present study, we found the $\Delta$ agr mutant strain having higher resistance to high concentrations of b-lactam antibiotics such as oxacillin and ampicillin. To determine the influence of variation in the microenvironment of cells between the parental and mutant strains, fatty acid analysis of the supernatant, total lipids, and phospholipid fatty acids were compared. The $\Delta$ agr mutant strain tended to produce fewer fatty acids and retained lower amounts of C16, C18 fatty acids in the supernatant. Phospholipid analysis showed a dramatic increase in the hydrophobic longerchain fatty acids in the membrane. To target membrane, we applied several surfactants and found that sorbitan monolaurate (Span20) had a synergistic effect with oxacillin by decreasing biofilm formation and growth. These findings indicate that agr deletion allows for MRSA to resist antibiotics via several changes including constant expression of mecA, fatty acid metabolism, and biofilm thickening.

\section{Introduction}

Staphylococcus aureus is a successful indigenous pathogen in humans, which expresses a wide range of virulence factors, including toxins, immune evasive surface factors, and virulent enzymes (Turner et al. 2019). Moreover, multi-resistant methicillin-resistant $S$. aureus (MRSA) has emerged as a major threat to human health. MRSA strains are generally classified into healthcare-associated MRSA (HA-MRSA) and community-associated MRSA (CA-MRSA), which differ with respect to several characteristics in addition to the source of infection (Mediavilla et al. 2012). HA-MRSA is commonly multi-drug resistant and occasionally causes pneumonia, urinary tract infection, and surgical site infection (Waness 2010). These strains pose a clinical challenge with respect to the difficulty in choice of an appropriate antibiotic for the treatment. In addition, MRSA does not produce the cytotoxin Panton-Valentine leukocidin, and contains type I, II, and III staphylococcal cassette chromosome mec. By contrast, CA-MRSA is resistant to $\beta$-lactam antibiotics, and causes skin and soft tissue infections along with necrotizing pneumonia (Asghar 2014). However, CA-MRSA strains tend to replicate at much faster rates than HA-MRSA strains, which facilitates their spread (Otter and French 2012). MRSA strains have acquired several mechanisms to inactivate or block the effects of antibiotics. For example, formation of a thick biofilm can act as a physical barrier to antibiotics, and structural differences have also evolved, including variations in membrane composition and modifications of the target of antibiotics (Boudjemaa et al. 2018; Craft et al. 2019; Fishovitz et al. 2014). Therefore, the antibiotic resistance of MRSA poses a significant treatment challenge and gaining greater insight into the mechanisms contributing to this resistance can help to guide treatment and control strategies. 
Accessory global regulator (Agr) is a main regulator of gram-positive bacteria (Cheung et al. 2011). In MRSA, the main function of Agr is to regulate biofilm formation and toxin production (Rutherford and Bassler 2012). The agroperon acquires signal molecules via a positively regulated mechanism so that RNAll (agrACDB) activates the production of phenol soluble modulins (PSMs) and the RNAll transcripts are also activated to produce $\delta$-hemolysin; moreover, the RNAlll transcript itself controls capsule formation, toxin production, and central metabolism of the strain (Kong et al. 2016).

PSMs are recently discovered short a-helical amphipathic peptides that cause neutrophil lysis after phagocytosis (Chatterjee et al. 2013; Otto 2014). In addition, PSMs have been shown to exert other functions, including acting as regulators in persister cells and biofilm formation (Periasamy et al. 2012; Xu et al. 2017). Following agr activation, PSMs are produced to develop the biofilm structure and disperse the biofilm by forming a complex with eDNA, extracellular polysaccharides, and proteins, or by taking advantage of its surfactant characteristics (Schilcher et al. 2016). Regulation of agr is also a trigger for changing the antibiotic resistance of MRSA. For example, agr expression is somewhat repressed in HAMRSA strains compared to that in CA-MRSA strains, whereas mecA (PBP2a), which has lower affinity to $\beta$-lactam, is highly expressed in HA-MRSA and hinders the efficacy of the single use of $\beta$-lactam antibiotics (Geisinger and Isberg 2017; Kaito et al. 2011; McCarthy et al. 2015). By contrast, agris highly expressed in CA-MRSA, which results in the production of more toxins rather than becoming resistant to antibiotics as is the case for HA-MRSA (Kaito et al. 2013). A recent study showed that mutation in the agr locus led to an increase in biofilm thickness, which might cause higher rates of antibiotic resistance (Periasamy et al. 2012). However, the detailed mechanism by which the agr system regulates the resistance mechanism remains unclear.

With the aim of elucidating this mechanism, in the present study, the $\beta$-lactam resistance of the agr mutant ( $\triangle a g r$ ) of the MRSA strain Staphylococcus aureus USA300-0114 was compared with that of the wild-type strain. Considering that various MRSA strains show stronger resistance with exposure to higher concentrations of antibiotics, we examined the influence of different concentrations of antibiotics that are typically used in microbiology experiments on resistance. To understand the nature of these differences, we further explored the differences in fatty acid distribution and membrane fatty acid composition between the wild-type (WT) and agr mutant strains to determine their influence on antibiotic resistance. These findings can provide new insight into the role of agrin resistance and guide new strategies for treatment of MRSA infections.

\section{Materials And Methods}

\section{Bacterial strains, media, and culture conditions}

For cell preparation, the wild-type strain Staphylococcus aureus USA300-0114 (LAC) (CDC. 2003) and the $\triangle$ agr mutant strain (Cheung et al. 2011) was cultured in tryptic soybean broth (TSB) agar and/or liquid broth. For pre-culture, a single colony of the strain from a TSB agar plate was used to inoculate $5 \mathrm{~mL}$ of TSB medium. Then, $1 \%(\mathrm{v} / \mathrm{v})$ of the cell culture suspension was inoculated in a 96 -well plate for antibiotic 
resistance test and cell cultivation was conducted for $24 \mathrm{~h}$ in incubator at $37^{\circ} \mathrm{C}$ without shaking unless stated otherwise.

\section{Antimicrobial susceptibility testing}

Test for antibiotic sensitivity was conducted by broth microdilution using 96-well plate. To set up MIC assay, 2-fold serial dilutions of oxacillin, gentamycin, erythromycin, vancomycin, chloramphenicol and teicoplanin were prepared in a microdilution plate. The inoculum was prepared from a sterile swab of colonies from an agar plate to make bacterial suspension which is equivalent to optical density $595 \mathrm{~nm}$ of $0.5 \mathrm{McF}$ arland standard. Then, the inoculum was dispensed to microdilution plate and incubated at 37 ${ }^{\circ} \mathrm{C}$ for $24 \mathrm{~h}$ without shaking.

\section{Analysis of cell growth and biofilm formation}

For analysis of cell growth and biofilm, $1 \%(\mathrm{v} / \mathrm{v})$ of the cell culture suspension from the pre-culture was inoculated in a 96-well plate and incubated at $37^{\circ} \mathrm{C}$ for $48 \mathrm{~h}$ without shaking. Cell growth was measured in terms of cell density, determined according to the optical density using a 96-well microplate reader (TECAN, Switzerland). Biofilm formation was analyzed with crystal violet staining using a previously reported method (Au - O'Toole 2011). Briefly, methanol fixation of biofilm was carried out and $200 \mu \mathrm{l}$ of $0.2 \%$ of crystal violet solution was added to each well and staining was conducted for 5 min for further analysis.

\section{Analysis of total fatty acids and fatty acids in the supernatant}

Gas chromatography-mass spectrometry (GC-MS) was used for the detection and quantification of total fatty acids and supernatant fatty acids, according to a previously described method with slight modification (Bhatia et al. 2017). For total fatty acids analysis, cell cultivation was conducted using $5 \mathrm{ml}$ of TSB with $1 \%(\mathrm{v} / \mathrm{v})$ inoculum in shaking incubator at $37^{\circ} \mathrm{C}$ and $200 \mathrm{rpm}$. Then, cell was collected at 24 $\mathrm{h}$ and $48 \mathrm{~h}$ by centrifugation at 3,000 $\mathrm{xg}$ for 20 min and washed twice with Milli Q water. The harvested cell was freeze-dried for further methanolysis to analyze total fatty acids. For the analysis of supernatant fatty acids in the medium, spent culture supernatant was incubated in shaking incubator for $2 \mathrm{~h}$ at $37{ }^{\circ} \mathrm{C}$ and $200 \mathrm{rpm}$ after adding $5 \mathrm{ml}$ of methanol and chloroform to extract fatty acids. The chloroform phase was collected and slowly evaporated under compressed $\mathrm{N}_{2}$ at $50{ }^{\circ} \mathrm{C}$ in heating block. Fatty acids were resolubilized with $1 \mathrm{ml}$ of chloroform for further steps. For methanolysis of fatty acids, approximately 10 $\mathrm{mg}$ of freeze-dried cells were weighed and placed in Teflon-stoppered glass vials, and then $1 \mathrm{~mL}$ chloroform and $1 \mathrm{~mL}$ methanol/ $\mathrm{H}_{2} \mathrm{SO}_{4}(85: 15 \mathrm{v} / \mathrm{v} \%)$ were added to the vials. After incubation at $100^{\circ} \mathrm{C}$ for $2 \mathrm{~h}$, the vials were cooled to room temperature, and then incubated on ice for $10 \mathrm{~min}$. After adding 1 
$\mathrm{mL}$ of ice-cold water, the samples were thoroughly mixed by vortexing for $1 \mathrm{~min}$ and then centrifuged at $3,000 \times \mathrm{g}$. The organic phases (bottom of the vials) were extracted by a pipette and transferred to clean borosilicate glass tubes containing $\mathrm{Na}_{2} \mathrm{SO}_{4}$. GC-MS was then performed with a Perkin Elmer Clarus 500 gas chromatograph that was connected to a Clarus $5 \mathrm{Q} 8 \mathrm{~S}$ mass spectrometer at $70 \mathrm{eV}(\mathrm{m} / \mathrm{z}$ 50-550; source at $230^{\circ} \mathrm{C}$ and quadruple at $\left.150{ }^{\circ} \mathrm{C}\right)$ in El mode with an Elite $5 \mathrm{MS}$ capillary column $(30 \mathrm{~m} \times 0.32$ $\mathrm{mm} \times 0.25 \mu \mathrm{m}$ film thickness; J\&W Scientific, USA). Helium was used as the carrier gas at a flow rate of $1.0 \mathrm{~mL} / \mathrm{min}$. The inlet temperature was maintained at $300^{\circ} \mathrm{C}$, and the oven was programmed to start at $150{ }^{\circ} \mathrm{C}$ for $2 \mathrm{~min}$ before increasing to $300^{\circ} \mathrm{C}$ at a rate of $4^{\circ} \mathrm{C} / \mathrm{min}$, and the temperature was maintained for $20 \mathrm{~min}$. The injection volume was $1 \mu \mathrm{L}$, with a split ratio of $50: 1$.

The structural assignments were based on interpretation of the mass spectrometric fragmentation and confirmed by comparison with the retention times and fragmentation patterns of the authentic compounds along with spectral data obtained from the online libraries of Wiley (http://www.palisade.com) and NIST (http://www.nist.gov). The internal standard was $1 \mu \mathrm{L}$ of methyl heneicosanoate $(10 \mathrm{mg} / \mathrm{mL}$ ) and Bacterial acid methyl ester (BAME) mix (Merck-Millipore, Burlington, MA, USA) was used to identify each peak of fatty acids and analytical standards for each fatty acid were used for quantification.

\section{Phospholipid fatty acid analysis}

For phospholipid analysis $10 \mathrm{~mL}$ of the liquid culture was carried out in shaking incubator using TSB with $1 \%(\mathrm{v} / \mathrm{v})$ inoculum at $37^{\circ} \mathrm{C}$ and $200 \mathrm{rpm}$. Samples were collected at $8 \mathrm{~h}$ and $16 \mathrm{~h}$ by centrifugation at $3,000 \mathrm{xg}$ for $20 \mathrm{~min}$, and cell pellet was suspended with $0.15 \mathrm{M}$ citric acid buffer/chloroform/methanol $(7: 7.5: 5 \mathrm{v} / \mathrm{v} / \mathrm{v})$ and incubated in the shaking incubator at $37^{\circ} \mathrm{C}$ and $200 \mathrm{rpm}$ for $2 \mathrm{~h}$ to extract total fatty acids. The chloroform phase was collected, and the chloroform was slowly evaporated under compressed $\mathrm{N}_{2}$ to avoid oxidation. The sample was loaded into a sialic column and elution was performed with $5 \mathrm{~mL}$ of chloroform, $5 \mathrm{~mL}$ of acetone, and $5 \mathrm{~mL}$ of methanol serially, and the methanol phase was collected for analysis of phospholipid fatty acids (PLFAs). One milliliter of toluene was added to the sample, which was subjected to mild alkaline trans-methylation with $1 \mathrm{~mL}$ of $\mathrm{KOH} / \mathrm{MeOH}$ at $37^{\circ} \mathrm{C}$ for $15 \mathrm{~min}$, followed by cooling to room temperature. A 2-mL aliquot of 4:1 n-hexane/chloroform was added, the sample was neutralized with $1 \mathrm{~mL}$ of $1 \mathrm{M}$ acetic acid, $2 \mathrm{~mL}$ of Milli $\mathrm{Q}$ water, and the phases were separated by centrifugation. The upper hexane layer was removed, and this step was repeated with fresh 2-mL aliquots of 4:1 n-hexane/chloroform. The combined hexane fractions were concentrated under compressed $\mathrm{N}_{2}$ and the fatty acids were re-solubilized with chloroform and analyzed using same method as described above for fatty acid analysis.

\section{Exogenous surfactants and fatty acids addition test}


Surfactants can be used for elimination of contaminating bacteria in medical devices (Hassan and Frank 2003). However, a surfactant itself is not bactericidal but rather modifies the permeability of the cell to be more susceptible to a disinfectant (Walton et al. 2008). Therefore, various surfactants with different properties, ranging from hydrophilic to hydrophobic, were screened to investigate the synergistic effect with antibiotic treatment (Table S1). Surfactants were solubilized in DMSO for the preparation of $10 \%$ $(\mathrm{v} / \mathrm{v})$ each stock solution. Pre-culture was conducted inoculating $5 \mathrm{ml}$ of TSB with a single colony from the agar plate, overnight in shaking incubator at $37^{\circ} \mathrm{C}$ and $200 \mathrm{rpm}$. Then, $1 \%(\mathrm{v} / \mathrm{v})$ of cell was inoculated into the 96 -well plate and incubated $48 \mathrm{~h}$ at $37^{\circ} \mathrm{C}$ without shaking for surfactant and fatty acid effect screening. Surfactants were added to each well at the final concentration of $0.1 \%(\mathrm{v} / \mathrm{v})$ using $10 \%(\mathrm{v} / \mathrm{v})$ stock solution for further investigation of their effects. To investigate the fatty acid effects, $100 \mu \mathrm{g} / \mathrm{ml}$ of fatty acids were added to each well as final concentrations using $10 \mathrm{mg} / \mathrm{ml}$ stocks in DMSO solution. Antibiotic treatment was carried out using $10 \mu \mathrm{g} / \mathrm{ml}$ and $100 \mu \mathrm{g} / \mathrm{ml}$ of oxacillin for the WT strain and $\Delta$ agrmutant strain according to their MIC value of oxacillin.

\section{Reverse transcription for CDNA synthesis and semi-quantitative real time PCR}

Pre-culture was conducted by inoculating $5 \mathrm{ml}$ of TSB with a single colony from agar plate, and incubating overnight in shaking incubator at $37^{\circ} \mathrm{C}$ and $200 \mathrm{rpm}$. Cell cultivations were carried out using 5 $\mathrm{ml}$ of TSB with $1 \%(\mathrm{v} / \mathrm{v})$ inoculum in shaking incubator at $37^{\circ} \mathrm{C}$ for $12 \mathrm{~h}$ and $24 \mathrm{~h}$ for RNA extraction. Cells were harvested using centrifuge at $3,000 \times \mathrm{g}$ for $20 \mathrm{~min}$. Then, total RNA was prepared using RNeasy Mini Kit (Qiagen, USA) and reverse transcription was performed with Superscript IV Reverse Transcriptase (Invitrogen Co., Carlsbad, CA) to generate the cDNA following the instructions manuals. Primer design was done using Primer express software v3.0.1 from Thermo Fisher Scientific (Waltham, MA, USA), and these primers can generate 150 bp PCR product for the comparison of gene expressions. Before semi-quantitative PCR, cycle number was optimized to set up the saturated gene expression level of $g y r B$ (endogenous control) for each template. After optimization, 25 cycle came out to be the optimal cycle number and further comparative analysis of gene expression became possible. Then, Semiquantitative PCR was conducted using LA taq with GC buffer I (Takara medical co. Itd) using the methods in manual. Primers used for the Semi-quantitative RT-PCR are listed in Table

S2.

\section{Results}

\section{Increased antibiotic resistance in the $\Delta$ agr mutant strain}

Cell growth was compared between the LAC (WT) strain and $\triangle$ agr mutant strain under exposure to high concentrations of oxacillin and ampicillin with the paper disc method on agar plate and antimicrobial susceptibility test with 96 -well plate. The $\Delta$ agr mutant strain was able to tolerate $1 \mu \mathrm{g}$ of ampicillin or oxacillin, whereas the wild-type strain was completely killed with only $0.5 \mu \mathrm{g}$ of oxacillin or ampicillin on agar plates (Fig. 1a, b). The $\Delta$ agr mutant strain was able to survive with even higher concentrations of the 
two antibiotics. Specifically, the $\Delta$ agr mutant strain could survive $50 \mu \mathrm{g} / \mathrm{ml}$ of oxacillin and $50 \mu \mathrm{g} / \mathrm{ml}$ of ampicillin. Antimicrobial susceptibility test have been conducted to see the difference in antibiotic resistance (Table 1). Especially, the $\Delta$ agr mutant was able to survive until oxacillin concentration reached $128 \mu \mathrm{g} / \mathrm{ml}$ but LAC (WT) strain did not grow when $32 \mu \mathrm{g} / \mathrm{ml}$ of oxacillin was used. For ampicillin, the $\triangle a g r$ mutant was not inhibited even when the concentration of ampicillin was $1082 \mu \mathrm{g} / \mathrm{ml}$ but LAC was inhibited at $60 \mu \mathrm{g} / \mathrm{ml}$ of ampicillin.

\section{Influence of agron total fatty acids, their distribution, and membrane composition}

GC-MS results showed that the amount of fatty acids in the $\Delta a g r$ mutant strain during $24 \mathrm{~h}$ and $48 \mathrm{~h}$ was lower than that of the WT strain. Among major fatty acids in the supernatant, 12-methyl-tetradecanoic acid (anteiso-C15:0), hexadecanoic acid (C16:0), and octadecanoic acid (C18:0) were decreased by the deletion of agr. A decrease in total amount of fatty acids was also recorded in the $\Delta$ agr mutant strain at $24 \mathrm{~h}$, though the difference was not significant between two strains at $48 \mathrm{~h}$ (Figure 2). Phospholipid analysis showed an increase in the portion of fatty acids with longer chains (Figure 3a, 3b). Moreover, the portion of 14-methyl-pentadecanoic acid (iso-C16:0), octadecanoic acid (C18:0), and eicosanoic acid (C20:0) in the cytoplasmic membrane were increased in the mutant strain. Long chain saturated fatty acids might have increased the survival rate of the mutant strain to $\beta$-lactam antibiotics by decreasing the membrane permeability and increasing the stability of the cytoplasmic membrane (Figure 3c) (Hashimoto and Hossain 2018; Uzman 2003).

\section{Influence of the exogenous addition of fatty acids on antibiotic resistance of MRSA}

Based on the analysis of fatty acids in the supernatant and phospholipid profiles, we further focused on the influence of $\mathrm{C} 16, \mathrm{C} 18$, and C20 fatty acids which were artificially added to the culture medium to verify the associated changes in antibiotic resistance. Only hexadecanoic acid (C16:0) had a significant anti-bacterial effect with or without oxacillin for both strains (Figure 4A). All the fatty acids added to the medium without oxacillin inhibited the biofilm formation but the effectiveness was minor with the single use of hexadecanoic acid (C16:0) in the $\Delta$ agr mutant strain (Figure $4 \mathrm{~B}$ ). When the $\Delta$ agr mutant strain was treated with oxacillin in the presence of fatty acids, biofilm formation was greatly reduced but the effect of synergy is not significant in the WT strain. This is because the WT strain has intact agroperon and responsiveness of synergism is dependent upon the concentration of oxacillin. One possible explanation is that the WT strain is sensitive to oxacillin and the growth is greatly limited so that the attenuated virulence tends to keep the biofilm formation to survive. Up to now, hexadecenoic acid (C16:0), octadecanoic acid (C18:0) and eicosanoic acid (C20:0) did not show a significant decrease in biofilm formation especially at low concentration (Lee et al. 2017). Hexadecenoic acid (C16:0) even increased the biofilm formation in MRSA strain whereas there was inhibition of biofilm formation with octadecanoic acid (C18:0) (Valliammai et al. 2019). However, hexadecenoic acid (C16:0), octadecanoic acid (C18:0) 
and eicosanoic acid (C20:0) could reduce biofilm formation in this study. This might be from the different culture condition including medium, bacterial strain and dose-dependent supplementation of fatty acid (Bravo-Santano et al. 2019a; Lade et al. 2019; Yoon et al. 2018). As a conclusion, this phenomenon might explain how the $\Delta$ agr mutant strain retained a lower level of fatty acid in the supernatant, since maintaining higher levels of fatty acids would interfere with biofilm formation and increase the instability of the cytoplasmic membrane leading to higher antibiotic susceptibility.

\section{Effect of surfactants on biofilm formation, membrane instability and antibiotic susceptibility}

Since the $\Delta$ agr mutant showed higher resistance to antibiotics along with the formation of a thicker biofilm and higher membrane integrity with long chain saturated fatty acids depending. The effect of surfactant on the susceptibility of MRSA to $\beta$-lactam antibiotics was studied. Although the bactericidal effects of the surfactants are not that significant, they can inhibit the cell growth of MRSA. The surfactant span20 has the greatest synergistic effect with oxacillin for both strains (Figure 5A). Inhibition of biofilm formation was dependent on the culture condition including the strain type, oxacillin and surfactants addition (Figure 5B), and span20 came out to be the most effective surfactant to eliminate biofilm. This difference might be related to differences in the chemical properties of the surfactants leading to the changes of membrane permeability and instability. Also, membrane microdomain is affected by membrane permeability or integrity so that span20 might have interfered with membrane microdomain assembly reducing the oligomerization of PBP2a thereby increasing antibiotic susceptibility (Garcia-Fernandez et al. 2017).

\section{Changes in mecA expression and the fatty acid synthetic operon without antibiotic treatment}

As mentioned above, HA-MRSA and CA-MRSA show differences in the expression levels of mecA and agr, which are major factors contributing to increased antibiotic resistance. Moreover, when considering the difference of fatty acid profiles, the expression level of the fatty acid synthetic operon in MRSA strains should be investigated. Therefore, the expression levels of $m e c A$, a regulator of fatty acid synthesis fapR and the rate limiting step of phospholipid synthesis $p / s X$ were compared between the LAC and $\triangle a g r$ mutant strains (Albanesi et al. 2013). Semi-quantitative RT-PCR showed that the mecA expression level decreased in the WT strain during cultivation but was constantly expressed in the agrmutant strain (Figure 6). In addition, although the fapR expression level is lower in mutant strain, the total amount of fatty acids was lower in the mutant strain by total fatty acid analysis. This might be from the fact that malonyl-coA concentration is not enough to interfere with the regulation effect of fapR on fatty acid metabolism. Also, it was found that there was lower expression of $p / s X$ in the $\Delta a g r$ mutant strain. As it is the rate-limiting step of phospholipid synthesis, the total amount of phospholipid decreased in the $\Delta a g r$ mutant strain (Figure S2). 


\section{Discussion}

MRSA is a group of gram-positive bacteria that are genetically distinct from other strains of $S$. aureus and represent a significant public health threat given the limited treatment options for these infections. Agr is a global regulator of MRSA with respect to toxin production, biofilm formation, and cell metabolism (Cheung et al. 2011). Specifically, agris activated to produce toxins increasing virulence during the cell growth and regulated by mecA expression when MRSA is exposed to $\beta$-lactam antibiotics. There is also a discrepancy between HA-MRSA and CA-MRSA with respect to mecA and agr expression (Waters et al. 2017), and the $\triangle$ agr mutant was shown to have a thicker biofilm compared to the WT strain LAC (Periasamy et al. 2012). Thus, we expected a variation of antibiotic resistance depending on the level of agr expression and conducted the present study to assess the antibiotic resistance of the agr deletion mutant. In addition, considering that various MRSA strains showed stronger resistance with higher concentration of antibiotics, we examined the antibiotic resistance of the mutant and WT strains with exposure to different concentrations of antibiotics that are typically used in microbiology experiments. Specifically, we tested high concentrations of $\beta$-lactam antibiotics $(>30 \mu \mathrm{g} / \mathrm{ml})$, at a level known to kill both methicillin-sensitive $S$. aureus and MRSA. Indeed, the mutant strain could survive at very high levels of the antibiotics, whereas the WT strain was killed at these same levels. Since the $\Delta$ agr mutant is derived from the LAC (WT) strain, this finding demonstrated that the deletion of agralone could increase the resistance to antibiotics such as oxacillin and ampicillin.

In MRSA, mec operon is generally inhibited by $m e c /$ repressing mecA expression to produce PBP2a which confers antibiotic resistance to MRSA. On the other hand, $\beta$-lactams increases mecA expression by binding to $m e c R 1$ which in turn cleaves the binding of mec/ onto the mec operon. However, USA300-0114 (LAC) strain contains SCCmec typelV which is a general type of CA-MRSA, and this type has mecRI mutation by insertion sequence. Therefore, the WT strain has constitutively low expression of mecA without antibiotic treatment (Rudkin et al. 2014). Along with the fact mentioned above, RT-PCR analysis showed that $m e c A$ was constantly expressed during cultivation in the $\Delta$ agr mutant strain increasing resistance to $\beta$-lactam antibiotics. In contrast, the WT strain has intact agr expression and had limited $m e c A$ expression at $24 \mathrm{~h}$ as is the case for general CA-MRSA strains. However, with deletion of agr, the expression level of $m e c A$ was constant and this suggests that agroperon regulate the expression of $m e c A$. Nevertheless, the antibiotic resistance mechanism is very complex and involves many more factors in addition to mecA expression. Therefore, we next focused on the potential mechanism contributing to the higher survival of the $\Delta$ agr mutant strain with exposure to much higher concentrations of antibiotics to understand the nature of this antibiotic resistance.

Additionally, we focused on fatty acid metabolism because a previous study showed that the agr mutation results in the lack of PSM production and a lower level of fatty acids secretion (Ebner et al. 2017). Additionally, innate immune system in epithelial cell and in abscesses take advantage of long chain free fatty acids such as linoleic acid [ C18:2 (n-6,9) ], oleic acid [ C18:1 (n-9) ] and palmitoleic acid [ $\mathrm{C} 16: 1$ (n-7) ], which are unsaturated fatty acids and saturated palmitic acid (C16:0) and stearic acid (C18:0) (Bravo-Santano et al. 2019b; Kenny et al. 2009). These major long chain fatty acids are 
considered bactericidal or bacteriostatic depending on their structural characteristics and they induce stress response of S.aureus. Fatty acids are known to have an antibacterial effect owing to their membrane-disrupting activity (Yoon et al. 2018). Therefore, the composition of total fatty acids and extracellular fatty acids were analyzed, and phospholipid fatty acid analysis was conducted with GC-MS. Overall, deletion of agr lower the amount of fatty acids in the supernatant and increase the content of long chain fatty acids in the cytoplasmic membrane. Maintaining lower amount of extracellular fatty acids can block the possible anti-bacterial effect from free fatty acids which interfere with electron transfer chain, protein assembly and increase membrane permeability and leakage (García-Fernández et al. 2017; Yoon et al. 2018). On the other hand, accumulation of long chain fatty acids decrease membrane permeability and increase membrane stability (Uzman 2003). These changes might have affected the antibiotic resistance of $\Delta$ agr mutant strain since physiology of phospholipid affect the antibiotic resistance (Rosado et al. 2015). Moreover, we showed span20 could affect the antibiotic susceptibility of MRSA in a complicated manner by changing membrane stability. With the changes in fatty acid profiles, mecA and fatty acid related genes and biofilm collectively led to the increased antibiotic resistance of MRSA. These findings further confirmed that agracts as a regulator for fatty acid metabolism, biofilm and $m e c A$ expression.

Overall, these results demonstrate the value in understanding the lipid synthesis and secretion of antibiotic-resistant bacteria as a complementary approach to the current focus on resistance genes such as $m e c A$, which can provide further guidance toward developing new strategies to overcome antibiotic resistance in MRSA.

\section{Declarations}

\section{Acknowledgements}

We thanks to Dr. Michael Otto. at Pathogen Molecular Genetics Section, Laboratory of Bacteriology, National Institute of Allergy and Infectious Diseases, U.S. National Institutes of Health, kindly giving us Staphylococcus aureus strains for our study. This work was supported by Research Program to solve social issues of the National Research Foundation of Korea (NRF) funded by the Ministry of Science ICT and Future Planning (2017M3A9E4077234) and National Research Foundation of Korea (NRF) (NRF2015M1A5A1037196, NRF-2019M3E6A1065160 and NRF-2019R1F1A1058805). In addition, this work was also supported by polar academic program (PAP, PE18900).

\section{Authors' contributions}

HS and YY conceived and designed the study. HS performed the experiments, and drafted the manuscript. TC, YH, YP, JP, SY, SB, RG, YK, JK and HJ interpreted the experimental results. HS, JK, HJ and $Y Y$ revised the manuscript. All authors read and approved the final manuscript. 


\section{Funding}

This work was supported by Research Program to solve social issues of the National Research Foundation of Korea (NRF) funded by the Ministry of Science ICT and Future Planning (2017M3A9E4077234) and National Research Foundation of Korea (NRF) (NRF-2015M1A5A1037196, NRF-2019M3E6A1065160 and NRF-2019R1F1A1058805). In addition, this work was also supported by polar academic program (PAP, PE18900).

\section{Availability of data and materials}

All the datasets on which the conclusions of the manuscript rely are presented in the main paper and additional information.

\section{Ethics approval and consent to participate}

This work does not contain any studies with human participants or animals.

\section{Consent for publication}

Not applicable.

\section{Competing interests}

The authors declare that they have no competing interests.

\section{References}

Albanesi D, Reh G, Guerin ME, Schaeffer F, Debarbouille M, Buschiazzo A, Schujman GE, de Mendoza D, Alzari PM (2013) Structural basis for feed-forward transcriptional regulation of membrane lipid homeostasis in Staphylococcus aureus. PLoS pathogens 9(1):e1003108 doi:10.1371/journal.ppat.1003108

Asghar AH (2014) Molecular characterization of methicillin-resistant Staphylococcus aureus isolated from tertiary care hospitals. Pak J Med Sci 30(4):698-702

Au - O'Toole GA (2011) Microtiter Dish Biofilm Formation Assay. JoVE(47):e2437 doi:doi:10.3791/2437 
Bhatia SK, Kim J, Song H-S, Kim HJ, Jeon J-M, Sathiyanarayanan G, Yoon J-J, Park K, Kim Y-G, Yang Y-H (2017) Microbial biodiesel production from oil palm biomass hydrolysate using marine Rhodococcus sp. YHY01. Bioresource Technology 233:99-109 doi:https://doi.org/10.1016/j.biortech.2017.02.061

Boudjemaa R, Cabriel C, Dubois-Brissonnet F, Bourg N, Dupuis G, Gruss A, Lévêque-Fort S, Briandet R, Fontaine-Aupart M-P, Steenkeste K (2018) Impact of Bacterial Membrane Fatty Acid Composition on the Failure of Daptomycin To Kill Staphylococcus aureus. Antimicrob Agents Chemother 62(7):e00023-18 doi:10.1128/aac.00023-18

Bravo-Santano N, Ellis J, Calle Y, Keun H, Behrends V, Letek M (2019a) Intracellular Staphylococcus aureus Elicits the Production of Host Very Long-Chain Saturated Fatty Acids with Antimicrobial Activity. Metabolites 9:148 doi:10.3390/metabo9070148

Bravo-Santano N, Ellis JK, Calle Y, Keun HC, Behrends V, Letek M (2019b) Intracellular Staphylococcus aureus Elicits the Production of Host Very Long-Chain Saturated Fatty Acids with Antimicrobial Activity. Metabolites 9(7):148 doi:10.3390/metabo9070148

CDC. (2003) Outbreaks of community-associated methicillin-resistant Staphylococcus aureus skin infections--Los Angeles County, California, 2002-2003. MMWR Morbidity and mortality weekly report 52(5):88

Chatterjee SS, Joo HS, Duong AC, Dieringer TD, Tan VY, Song Y, Fischer ER, Cheung GY, Li M, Otto M (2013) Essential Staphylococcus aureus toxin export system. Nature medicine 19(3):364-7 doi:10.1038/nm.3047

Cheung GYC, Wang R, Khan BA, Sturdevant DE, Otto M (2011) Role of the accessory gene regulator agr in community-associated methicillin-resistant Staphylococcus aureus pathogenesis. Infect Immun 79(5):1927-1935 doi:10.1128/iai.00046-11

Craft KM, Nguyen JM, Berg LJ, Townsend SD (2019) Methicillin-resistant Staphylococcus aureus (MRSA): antibiotic-resistance and the biofilm phenotype. MedChemComm 10(8):1231-1241 doi:10.1039/c9md00044e

Ebner P, Luqman A, Reichert S, Hauf K, Popella P, Forchhammer K, Otto M, Götz F (2017) Non-classical Protein Excretion Is Boosted by PSMa-Induced Cell Leakage. Cell Rep 20(6):1278-1286 doi:10.1016/j.celrep.2017.07.045

Fishovitz J, Hermoso JA, Chang M, Mobashery S (2014) Penicillin-binding protein 2a of methicillinresistant Staphylococcus aureus. IUBMB Life 66(8):572-577 doi:10.1002/iub.1289

García-Fernández E, Koch G, Wagner R, Fekete A, Stengel S, Schneider J, Mielich-Süß B, Geibel S, Markert S, Stigloher C, Lopez D (2017) Membrane Microdomain Disassembly Inhibits MRSA Antibiotic Resistance. Cell 171 doi:10.1016/j.cell.2017.10.012 
Geisinger E, Isberg RR (2017) Interplay Between Antibiotic Resistance and Virulence During Disease Promoted by Multidrug-Resistant Bacteria. J Infect Dis 215(suppl_1):S9-S17 doi:10.1093/infdis/jiw402

Hashimoto M, Hossain S (2018) Fatty Acids: From Membrane Ingredients to Signaling Molecules.

Hassan AN, Frank JF (2003) Influence of surfactant hydrophobicity on the detachment of Escherichia coli 0157:H7 from lettuce. International journal of food microbiology 87(1-2):145-52

Kaito C, Saito Y, Ikuo M, Omae Y, Mao H, Nagano G, Fujiyuki T, Numata S, Han X, Obata K, Hasegawa S, Yamaguchi H, Inokuchi K, Ito T, Hiramatsu K, Sekimizu K (2013) Mobile genetic element SCCmec-encoded psm-mec RNA suppresses translation of agrA and attenuates MRSA virulence. PLoS pathogens 9(4):e1003269 doi:10.1371/journal.ppat.1003269

Kaito C, Saito Y, Nagano G, Ikuo M, Omae Y, Hanada Y, Han X, Kuwahara-Arai K, Hishinuma T, Baba T, Ito T, Hiramatsu K, Sekimizu K (2011) Transcription and translation products of the cytolysin gene psm-mec on the mobile genetic element SCCmec regulate Staphylococcus aureus virulence. PLoS pathogens 7(2):e1001267 doi:10.1371/journal.ppat.1001267

Kenny JG, Ward D, Josefsson E, Jonsson I-M, Hinds J, Rees HH, Lindsay JA, Tarkowski A, Horsburgh MJ (2009) The Staphylococcus aureus Response to Unsaturated Long Chain Free Fatty Acids: Survival Mechanisms and Virulence Implications. PloS one 4(2):e4344 doi:10.1371/journal.pone.0004344

Kong C, Neoh HM, Nathan S (2016) Targeting Staphylococcus aureus Toxins: A Potential form of AntiVirulence Therapy. Toxins 8(3) doi:10.3390/toxins8030072

Lade H, Park J, Chung S, Kim I, Kim J-M, Joo H-S, Kim J-S (2019) Biofilm Formation by Staphylococcus aureus Clinical Isolates is Differentially Affected by Glucose and Sodium Chloride Supplemented Culture Media. Journal of clinical medicine 8 doi:10.3390/jcm8111853

Lee J-H, Kim Y-G, Park J, Lee J (2017) Supercritical fluid extracts of Moringa oleifera and their unsaturated fatty acid components inhibit biofilm formation by Staphylococcus aureus. Food Control 80 doi:10.1016/j.foodcont.2017.04.035

McCarthy H, Rudkin JK, Black NS, Gallagher L, O'Neill E, O'Gara JP (2015) Methicillin resistance and the biofilm phenotype in Staphylococcus aureus. Frontiers in cellular and infection microbiology 5:1 doi:10.3389/fcimb.2015.00001

Mediavilla JR, Chen L, Mathema B, Kreiswirth BN (2012) Global epidemiology of community-associated methicillin resistant Staphylococcus aureus (CA-MRSA). Current opinion in microbiology 15(5):588-95 doi:10.1016/j.mib.2012.08.003

Otter JA, French GL (2012) Community-associated meticillin-resistant <em>Staphylococcus aureus</em>: the case for a genotypic definition. Journal of Hospital Infection 81(3):143-148 doi:10.1016/j.jhin.2012.04.009

Page 14/21 
Otto M (2014) Phenol-soluble modulins. Int J Med Microbiol 304(2):164-169 doi:10.1016/j.ijmm.2013.11.019

Periasamy S, Joo H-S, Duong AC, Bach T-HL, Tan VY, Chatterjee SS, Cheung GYC, Otto M (2012) How \&lt;em\&gt;Staphylococcus aureus\&lt;/em\&gt; biofilms develop their characteristic structure. Proceedings of the National Academy of Sciences 109(4):1281 doi:10.1073/pnas.1115006109

Rosado H, Turner RD, Foster SJ, Taylor PW (2015) Impact of the beta-Lactam Resistance Modifier (-)Epicatechin Gallate on the Non-Random Distribution of Phospholipids across the Cytoplasmic Membrane of Staphylococcus aureus. Int J Mol Sci 16(8):16710-16727 doi:10.3390/ijms160816710

Rudkin JK, Laabei M, Edwards AM, Joo H-S, Otto M, Lennon KL, O'Gara JP, Waterfield NR, Massey RC (2014) Oxacillin alters the toxin expression profile of community-associated methicillin-resistant Staphylococcus aureus. Antimicrob Agents Chemother 58(2):1100-1107 doi:10.1128/aac.01618-13

Rutherford ST, Bassler BL (2012) Bacterial quorum sensing: its role in virulence and possibilities for its control. Cold Spring Harbor perspectives in medicine 2(11) doi:10.1101/cshperspect.a012427

Schilcher K, Andreoni F, Dengler Haunreiter V, Seidl K, Hasse B, Zinkernagel AS (2016) Modulation of Staphylococcus aureus Biofilm Matrix by Subinhibitory Concentrations of Clindamycin. Antimicrob Agents Chemother 60(10):5957-67 doi:10.1128/AAC.00463-16

Turner NA, Sharma-Kuinkel BK, Maskarinec SA, Eichenberger EM, Shah PP, Carugati M, Holland TL, Fowler VG, Jr. (2019) Methicillin-resistant Staphylococcus aureus: an overview of basic and clinical research. Nature reviews Microbiology 17(4):203-218 doi:10.1038/s41579-018-0147-4

Uzman A (2003) Molecular biology of the cell (4th ed.): Alberts, B., Johnson, A., Lewis, J., Raff, M., Roberts, K., and Walter, P. Biochemistry and Molecular Biology Education 31:212-214 doi:10.1002/bmb.2003.494031049999

Valliammai A, Sethupathy S, Priya A, Selvaraj A, Bhaskar JP, Krishnan V, Pandian SK (2019) 5Dodecanolide interferes with biofilm formation and reduces the virulence of Methicillin-resistant Staphylococcus aureus (MRSA) through up regulation of agr system. Scientific reports 9(1):13744 doi:10.1038/s41598-019-50207-y

Walton JT, Hill DJ, Protheroe RG, Nevill A, Gibson H (2008) Investigation into the effect of detergents on disinfectant susceptibility of attached Escherichia coli and Listeria monocytogenes. Journal of Applied Microbiology 105(1):309-315 doi:10.1111/j.1365-2672.2008.03805.x

Waness A (2010) Revisiting Methicillin-Resistant Staphylococcus aureus Infections. J Glob Infect Dis 2(1):49-56 doi:10.4103/0974-777x.59251

Waters EM, Rudkin JK, Coughlan S, Clair GC, Adkins JN, Gore S, Xia G, Black NS, Downing T, O'Neill E, Kadioglu A, O'Gara JP (2017) Redeploying $\beta$-Lactam Antibiotics as a Novel Antivirulence Strategy for the 
Treatment of Methicillin-Resistant Staphylococcus aureus Infections. J Infect Dis 215(1):80-87 doi:10.1093/infdis/jiw461

Xu T, Wang X-Y, Cui P, Zhang Y-M, Zhang W-H, Zhang Y (2017) The Agr Quorum Sensing System Represses Persister Formation through Regulation of Phenol Soluble Modulins in Staphylococcus aureus. Front Microbiol 8:2189-2189 doi:10.3389/fmicb.2017.02189

Yoon BK, Jackman JA, Valle-González ER, Cho N-J (2018) Antibacterial Free Fatty Acids and Monoglycerides: Biological Activities, Experimental Testing, and Therapeutic Applications. Int J Mol Sci 19(4):1114 doi:10.3390/ijms19041114

\section{Table}

Table 1. Antibiotic susceptibility test for LAC WT and LAC $\Delta$ agr strains.

\begin{tabular}{|c|c|c|c|c|c|c|}
\hline \multirow[t]{3}{*}{ Antibiotics } & \multirow{3}{*}{$\begin{array}{l}\text { Disk } \\
\text { Content }\end{array}$} & \multirow{2}{*}{\multicolumn{2}{|c|}{$\begin{array}{l}\text { Disk diffusion } \\
\text { Zone Diameter (mm) }\end{array}$}} & \multirow[t]{3}{*}{ Range } & \multirow{2}{*}{\multicolumn{2}{|c|}{$\begin{array}{l}\text { Broth microdilution } \\
\text { MIC }(\mu \mathrm{g} / \mathrm{ml})\end{array}$}} \\
\hline & & & & & & \\
\hline & & $\begin{array}{l}\text { LAC } \\
\text { WT }\end{array}$ & $\begin{array}{l}\text { LAC } \\
\Delta a g r\end{array}$ & & LAC WT & $\overline{\mathrm{LAC}} \Delta \mathrm{agr}$ \\
\hline Cefoxitin & $30 \mu \mathrm{g}$ & $13(\mathrm{R})$ & $13(\mathrm{R})$ & $0.5-256$ & $>32(\mathrm{R})$ & $>64(\mathrm{R})$ \\
\hline Teicoplanin & $30 \mu g$ & $16(\mathrm{~S})$ & $15(\mathrm{~S})$ & $1.5-24$ & $3(\mathrm{~S})$ & $6(\mathrm{~S})$ \\
\hline Gentamicin & $10 \mu g$ & $25.6(\mathrm{~S})$ & $20(S)$ & $0.5-8$ & $4(S)$ & $8(\mathrm{I})$ \\
\hline Erythromycin & $15 \mu g$ & $29(\mathrm{~S})$ & $10(\mathrm{~S})$ & $\begin{array}{l}0.25- \\
128\end{array}$ & $\begin{array}{l}0.25 \\
\text { (R) }\end{array}$ & $>64(\mathrm{R})$ \\
\hline Chloramphenicol & $30 \mu \mathrm{g}$ & $22(\mathrm{~S})$ & $22(\mathrm{R})$ & $3-48$ & $12(\mathrm{I})$ & $12(\mathrm{I})$ \\
\hline Vancomycin & N.I & N.I & N.I & $0.5-8$ & $1(\mathrm{~S})$ & $4(\mathrm{I})$ \\
\hline Oxacillin & N.I & N.I & N.I & $8-256$ & $>32(\mathrm{R})$ & $>128(\mathrm{R})$ \\
\hline Ampicillin & N.I & N.I & N.I & $16-1082$ & $>64(\mathrm{R})$ & $\begin{array}{l}>1028 \\
(\mathrm{R})\end{array}$ \\
\hline
\end{tabular}

\section{Figures}


Fig. 1

A

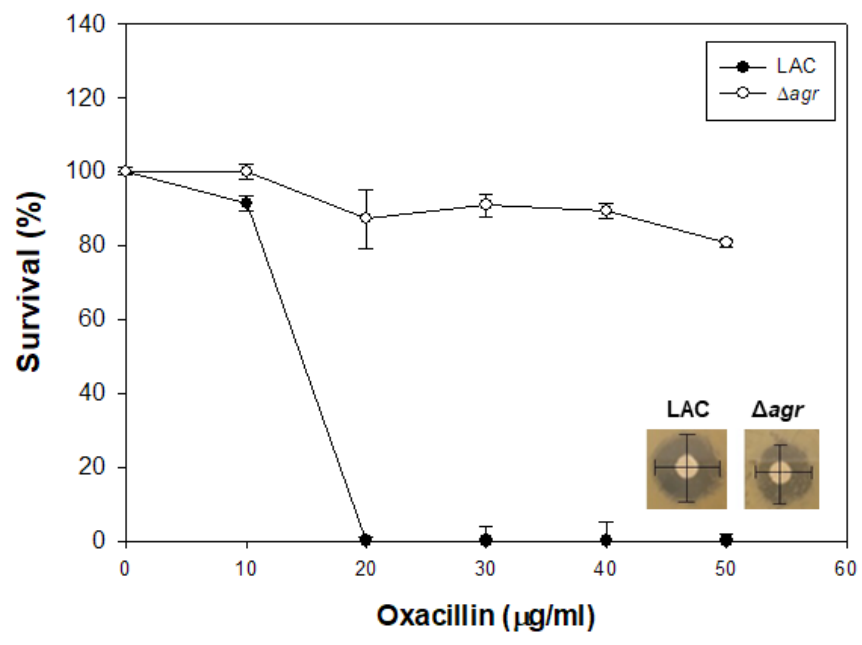

B

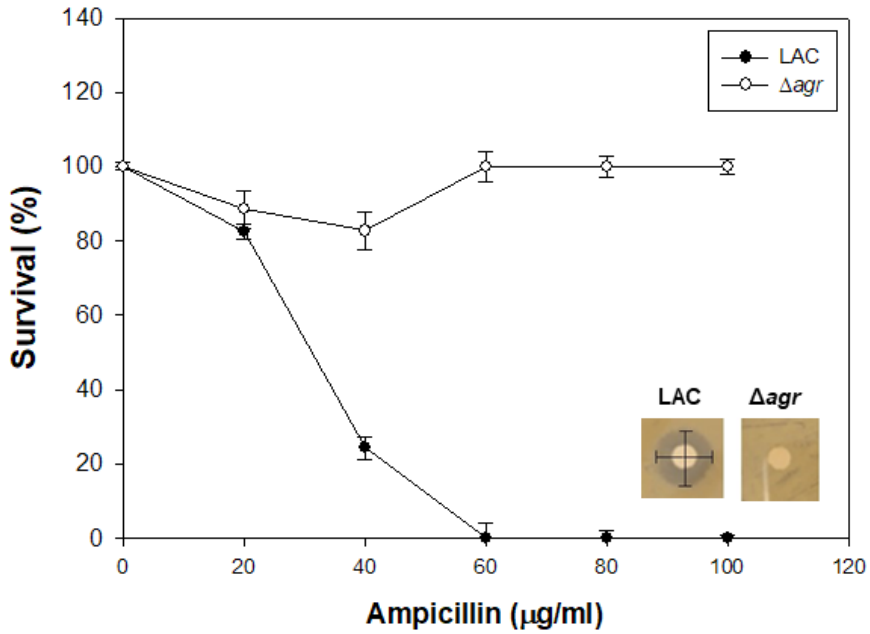

\section{Figure 1}

Antibiotic susceptibility test of the LAC and Dagr mutant strain with oxacillin and ampicillin. In the presence of $50 \mu \mathrm{g} / \mathrm{ml}$ of oxacillin and ampicillin the disk diffusion method was carried out. The error bars represent the standard deviation of three replicates 
Fig. 2

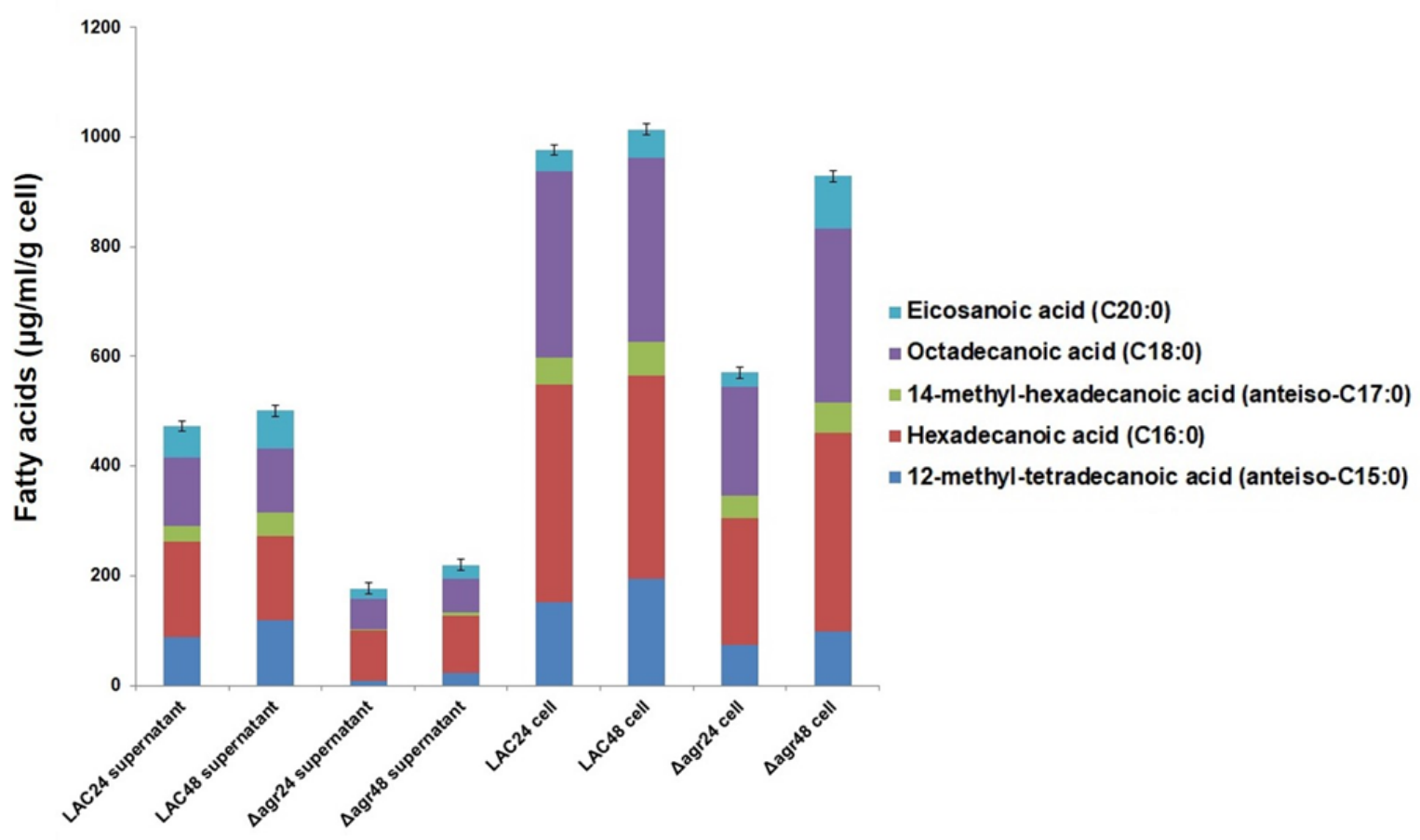

Figure 2

Comparison of total fatty acids and supernatant fatty acid profiles between the LAC and Dagr mutant strain. Supernatant and cell biomass samples were taken separately at $24 \mathrm{~h}$ and $48 \mathrm{~h} .24$ and 48 following strain name indicate the time point of sample collected. The error bars represent the standard deviation of three replicates

Fig. 3
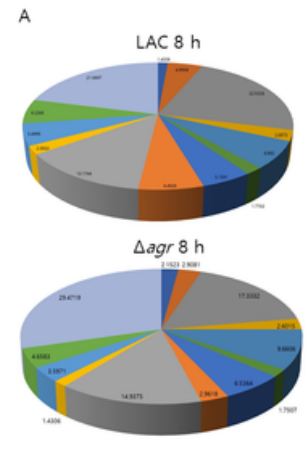
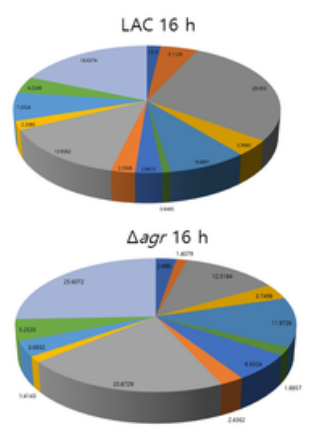

- 12-methyl-tridecanoic acid (iso-C14:0) - Pentadecanoic acid (C15:0) "12-methyl-tetradecanoic acid (anteiso-C15:0) - Hexadecanoic acid (C16:0) 14-methyl-pentadecanoic acid (iso-c16:0) 15-methyl-hexadecanoic acid (iso-c17:0) 16-methyl-heptadecanoic acid (iso.C18:0) Octadecanoic acid (c18:0) -17-methyl-octadecanoic acid (iso-C19:0) =16-methyl-octadecanoic acid (anteiso-C19:0) "Nonadecanoic acid (C19:0)
-Eicosanoic acid (C20:0)

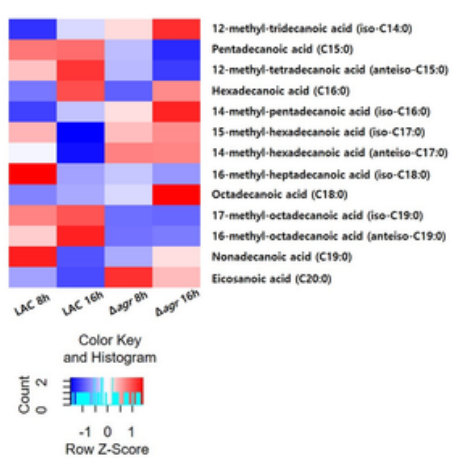

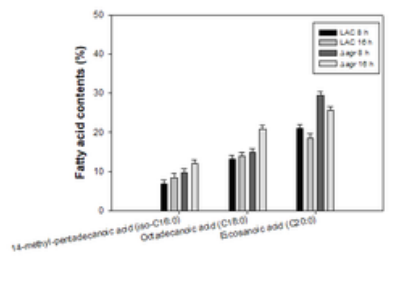

Figure 3

Comparison of phospholipid fatty acids in the LAC and $\triangle$ agr mutant strains. (a) Compositional analysis of membrane fatty acids at different time points shown as a pie chart. (b) Compositional analysis of membrane fatty acids at different time points shown as a heatmap. (c) Fatty acid contents of 14-methyl- 
pentadecanoic acid (iso-C16:0) and Octadecanoic acid (C18:0) in MRSA. The error bars represent the standard deviation of three replicates

Fig. 4

A

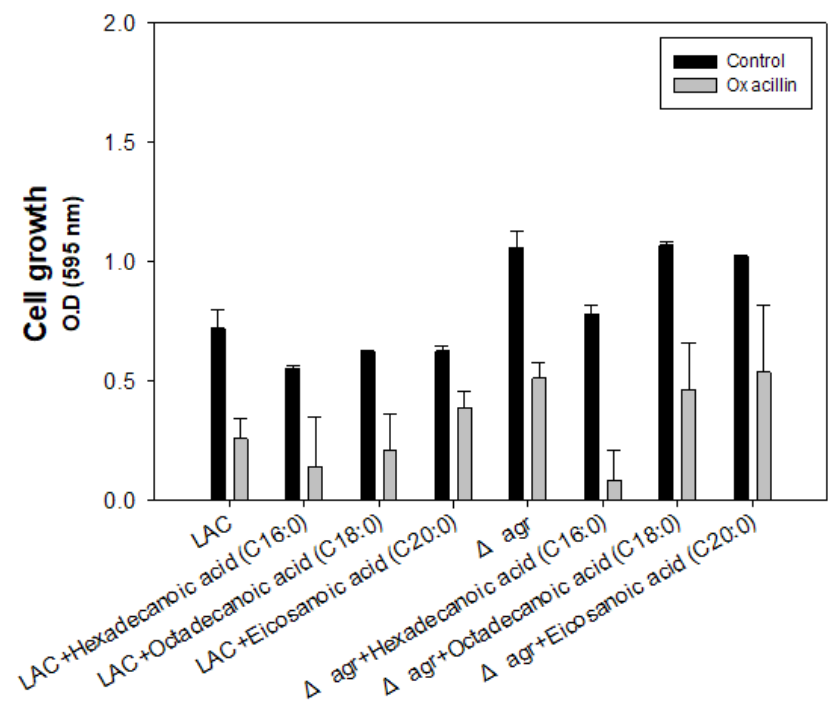

B

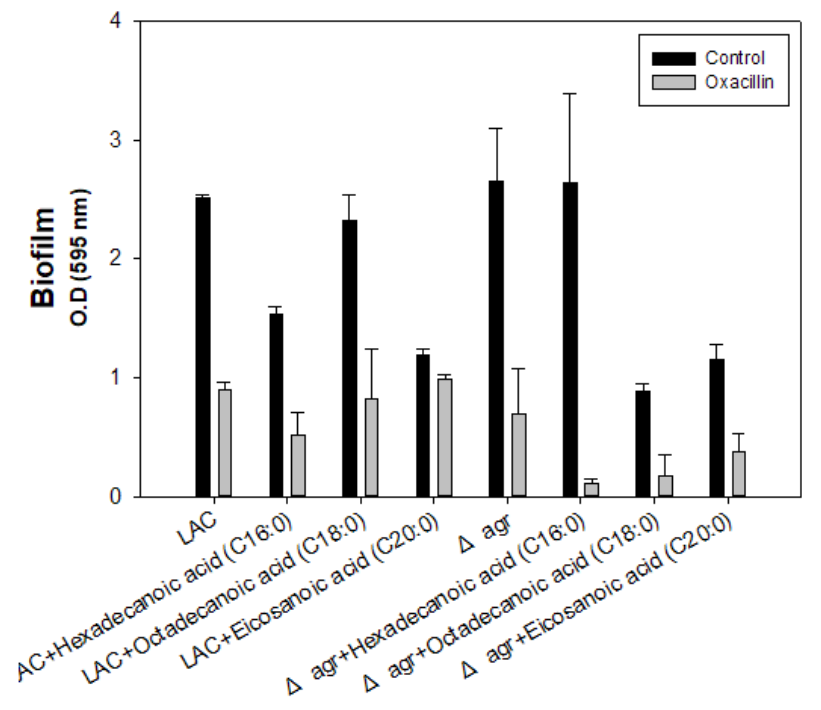

Figure 4

Synergistic effect of oxacillin with artificially added fatty acids. (a) Cell growth of the LAC and $\square$ agr mutant strains with the addition of long-chain fatty acids. (b) Biofilm formation of the LAC and Dagr mutant strains with long-chain fatty acids. The cell was grown for $48 \mathrm{~h}$ in the presence of $100 \mu \mathrm{g} / \mathrm{ml}$ of the fatty acid supplements for the comparative analysis of cell growth and biofilm formation. The error bars represent the standard deviation of three replicates 
Fig. 5

A

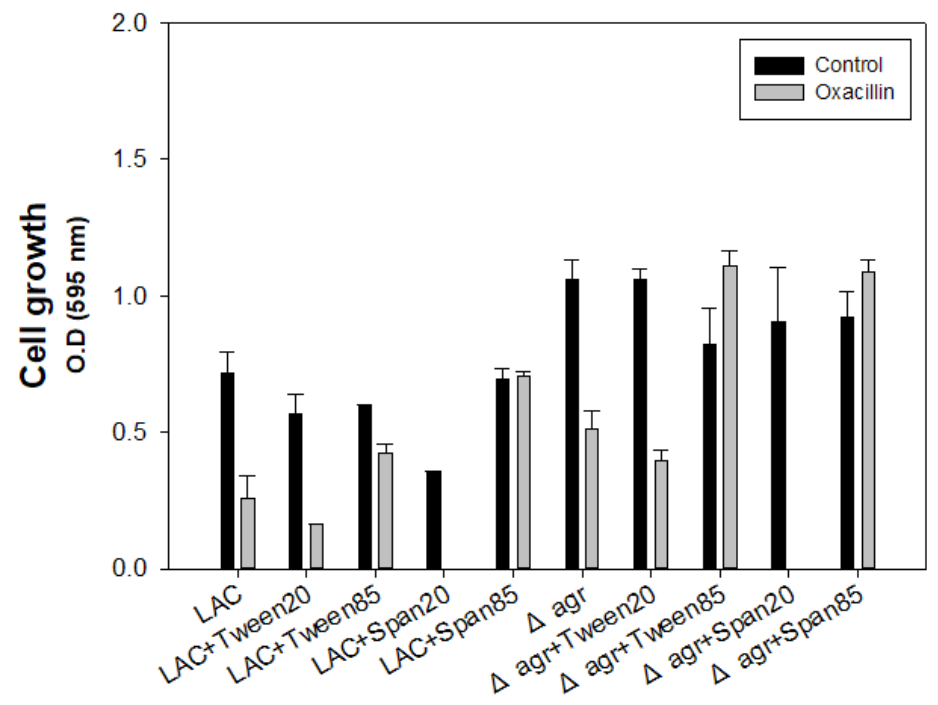

B

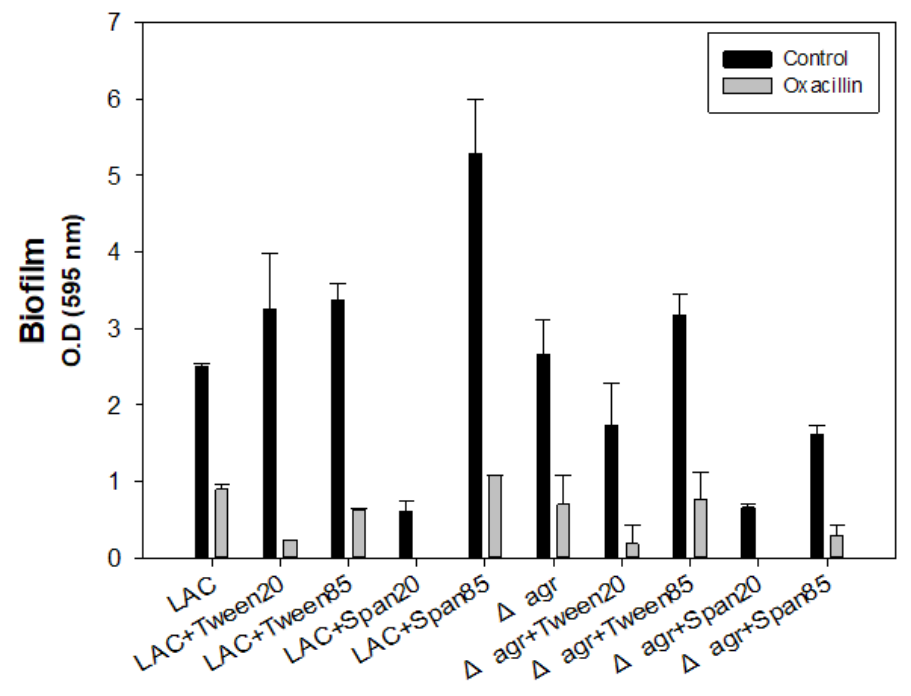

Figure 5

Synergistic effect of surfactants and oxacillin. (a) Cell growth of the LAC and Dagr mutant strains with surfactants. (b) Biofilm formation of the LAC and Dagr mutant strains with surfactants. The cell was grown for $48 \mathrm{~h}$ in the presence of $0.1 \%(\mathrm{v} / \mathrm{v})$ of the surfactant supplements for the comparative analysis of cell growth and biofilm formation. The error bars represent the standard deviation of three replicates 
Fig. 6

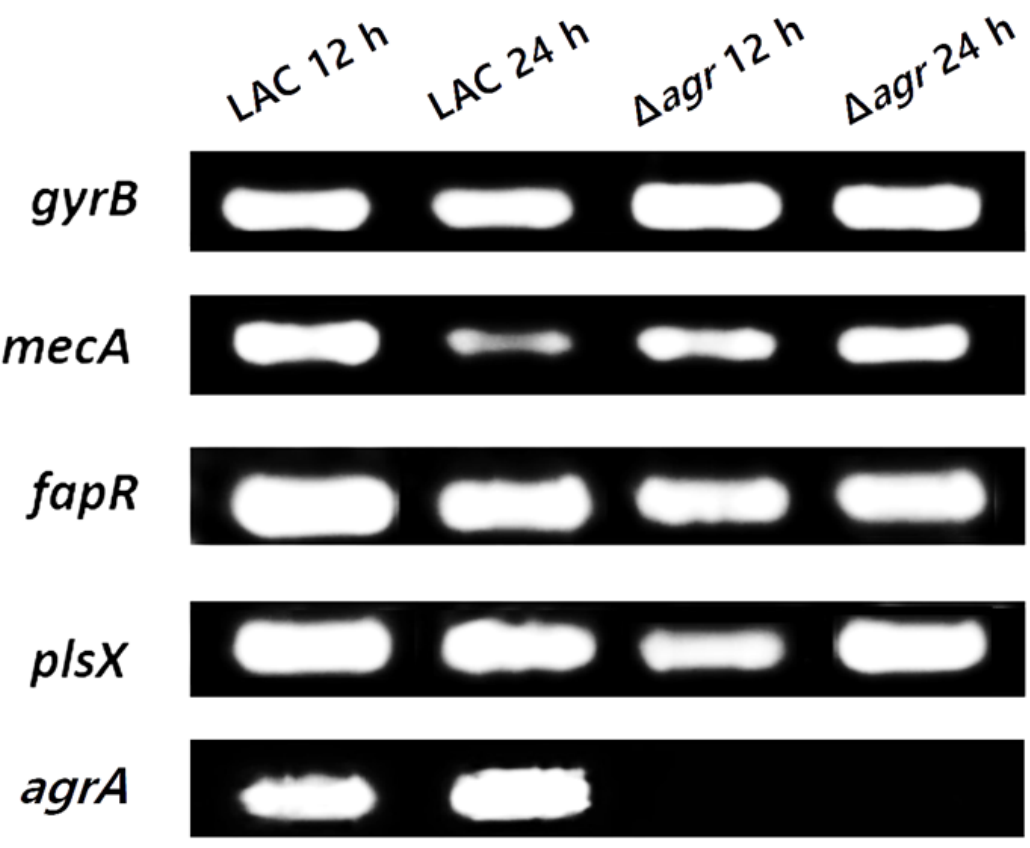

Figure 6

Semi-quantitative RT-PCR of mecA and the fatty acid synthetic operon.

\section{Supplementary Files}

This is a list of supplementary files associated with this preprint. Click to download.

- AMBED1900333SupplementaryMaterials20.3.24.docx 